International Journal of Pure and Applied Mathematics

Volume 92 No. 5 2014, 703-718

ISSN: 1311-8080 (printed version); ISSN: 1314-3395 (on-line version)

url: http://www.ijpam.eu

doi: http://dx.doi.org/10.12732/ijpam.v92i5.6

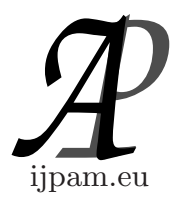

\title{
JOIN PRESERVING MAPS, FUZZY PREORDERS AND ALEXANDROV FUZZY TOPOLOGIES
}

\author{
Yong Chan Kim \\ Department of Mathematics \\ Gangneung-Wonju University \\ Gangneung, Gangwondo, 210-702, KOREA
}

\begin{abstract}
In this paper, we investigate the properties of join preserving maps in complete residuated lattices. We define join approximation operators as a generalization of fuzzy rough sets in complete residuated lattices. Moreover, we investigate the relations between join preserving operators and Alexandrov fuzzy topologies. We give their examples.
\end{abstract}

AMS Subject Classification: 03E72, 03G10, 06A15, 06F07

Key Words: complete residuated lattices, fuzzy preorders, join, meet, join preserving maps, join approximation operators, Alexandrov fuzzy topologies

\section{Introduction}

Pawlak $[9,10]$ introduced the rough set theory as a formal tool to deal with imprecision and uncertainty in the data analysis. The relationship between rough set theory and topological spaces was investigated in sets [6], on left-continuous t-norm [12]. Hájek [2] introduced a complete residuated lattice which is an algebraic structure for many valued logic. It is an important mathematical tool for algebraic structure of fuzzy contexts [1-5, 7,8, 11-16]. By using the concepts of lower and upper approximation operators, information systems and decision rules are investigated in complete residuated lattices $[1,2,7,8,11,12]$. Kim [5] investigated between fuzzy rough set and fuzzy quasi-uniform spaces in complete residuated lattices. Bělohlávek [1] developed the notion of fuzzy contexts using

Received: February 28, 2014

(c) 2014 Academic Publications, Ltd. url: www.acadpubl.eu 
Galois connections with $R \in L^{X \times Y}$ on a complete residuated lattice. Zhang $[13,14]$ introduced the fuzzy complete lattice which is defined by join and meet on fuzzy posets. Kim [4] investigated the properties of join (resp. meet, meet join, join meet) preserving operators in complete residuated lattices.

In this paper, we investigate the properties of join preserving maps in complete residuated lattice. By using the concepts of the fuzzy complete lattices $[13,14]$, we generalize upper approximation operators without fuzzy relations in complete residuated lattices. Moreover, we investigate the relations between join preserving maps and Alexandrov fuzzy topologies. We give their examples.

\section{Preliminaries}

Definition 2.1. $[1,2]$ A structure $(L, \vee, \wedge, \odot, \rightarrow, \perp, \top)$ is called a complete residuated lattice iff it satisfies the following properties:

(L1) $(L, \vee, \wedge, \perp, \top)$ is a complete lattice where $\perp$ is the bottom element and $\top$ is the top element;

(L2) $(L, \odot, \top)$ is a monoid;

(L3) adjointness properties hold,i.e.

$$
x \leq y \rightarrow z \text { iff } x \odot y \leq z .
$$

A operator ${ }^{*}: L \rightarrow L$ defined by $a^{*}=a \rightarrow \perp$ is called strong negations if $a^{* *}=a$.

$$
\top_{x}(y)=\left\{\begin{array}{ll}
\top, & \text { if } y=x, \\
\perp, & \text { otherwise. }
\end{array} \top_{x}^{*}(y)= \begin{cases}\perp, & \text { if } y=x, \\
\top, & \text { otherwise. }\end{cases}\right.
$$

In this paper, we assume that $\left(L, \vee, \wedge, \odot, \rightarrow,^{*}, \perp, \top\right)$ be a complete residuated lattice with a strong negation *

Definition 2.2. [13,14] Let $X$ be a set. A function $e_{X}: X \times X \rightarrow L$ is called:

(E1) reflexive if $e_{X}(x, x)=1$ for all $x \in X$,

(E2) transitive if $e_{X}(x, y) \odot e_{X}(y, z) \leq e_{X}(x, z)$, for all $x, y, z \in X$,

(E3) if $e_{X}(x, y)=e_{X}(y, x)=1$, then $x=y$.

If $e$ satisfies $(\mathrm{E} 1)$ and $(\mathrm{E} 2),\left(X, e_{X}\right)$ is a fuzzy preorder set. If $e$ satisfies (E1), (E2) and (E3), ( $\left.X, e_{X}\right)$ is a fuzzy partially order set (simply, fuzzy poset).

Example 2.3. (1) We define a function $e_{L}: L \times L \rightarrow L$ as $e_{L}(x, y)=x \rightarrow y$. Then $\left(L, e_{L}\right)$ is a fuzzy poset. 
(2) We define a function $e_{L^{X}}: L^{X} \times L^{X} \rightarrow L$ as $e_{L^{X}}(A, B)=\bigwedge_{x \in X}(A(x) \rightarrow$ $B(x))$. Then $\left(L^{X}, e_{L^{X}}\right)$ is a fuzzy poset from Lemma $2.10(9)$.

Definition 2.4. $[13,14]$ Let $\left(X, e_{X}\right)$ be a fuzzy poset and $A \in L^{X}$.

(1) A point $x_{0}$ is called a join of $A$, denoted by $x_{0}=\sqcup A$, if it satisfies (J1) $A(x) \leq e_{X}\left(x, x_{0}\right)$,

$(\mathrm{J} 2) \bigwedge_{x \in X}\left(A(x) \rightarrow e_{X}(x, y)\right) \leq e_{X}\left(x_{0}, y\right)$.

A point $x_{1}$ is called a meet of $A$, denoted by $x_{1}=\sqcap A$, if it satisfies

(M1) $A(x) \leq e_{X}\left(x_{1}, x\right)$,

(M2) $\bigwedge_{x \in X}\left(A(x) \rightarrow e_{X}(y, x)\right) \leq e_{X}\left(y, x_{1}\right)$.

Remark 2.5. [13,14] Let $\left(X, e_{X}\right)$ be a fuzzy poset and $A \in L^{X}$.

(1) If $x_{0}$ is a join of $A$, then it is unique because $e_{X}\left(x_{0}, y\right)=e_{X}\left(y_{0}, y\right)$ for all $y \in X$, put $y=x_{0}$ or $y=y_{0}$, then $e_{X}\left(x_{0}, y_{0}\right)=e_{X}\left(y_{0}, x_{0}\right)=\top$ implies $x_{0}=y_{0}$. Similarly, if a meet of $A$ exists, then it is unique.

(2) $x_{0}$ is a join of $A$ iff $\bigwedge_{x \in X}\left(A(x) \rightarrow e_{X}(x, y)\right)=e_{X}\left(x_{0}, y\right)$.

(3) $x_{1}$ is a meet of $A$ iff $\bigwedge_{x \in X}\left(A(x) \rightarrow e_{X}(y, x)\right)=e_{X}\left(y, x_{1}\right)$.

Remark 2.6. $[13,14]$ Let $\left(L, e_{L}\right)$ be a fuzzy poset and $A \in L^{L}$.

(1) Since $x_{0}$ is a join of $A$ iff $\bigwedge_{x \in L}\left(A(x) \rightarrow e_{L}(x, y)\right)=\bigwedge_{x \in L}(A(x) \rightarrow$ $(x \Rightarrow y))=\bigvee_{x \in L}(x \odot A(x)) \rightarrow y=e_{L}\left(x_{0}, y\right)=x_{0} \rightarrow y$, then $x_{0}=\sqcup A=$ $\bigvee_{x \in L}(x \odot A(x))$.

(2) Since $x_{0}$ is a join of $A$ iff $\bigwedge_{x \in L}\left(A(x) \rightarrow e_{L}(x, y)=\bigwedge_{x \in L}(A(x) \rightarrow(y \rightarrow\right.$ $x))=\bigwedge_{x \in L}(y \rightarrow(A(x) \rightarrow x))=y \rightarrow \bigwedge_{x \in L}(A(x) \rightarrow x)=y \rightarrow \sqcap A$, then $\sqcap A=\bigwedge_{x \in L}(A(x) \rightarrow x)$.

Remark 2.7. $[13,14]$ Let $\left(L^{X}, e_{L^{X}}\right)$ be a fuzzy poset and $\Phi \in L^{L^{X}}$.

(1) We have $\sqcup \Phi=\bigvee_{A \in L^{X}}(\Phi(A) \odot A)$ from:

$$
\begin{aligned}
& \bigwedge_{A \in L^{X}}\left(\Phi(A) \rightarrow e_{L^{X}}(A, B)\right) \\
& =e_{L^{X}}\left(\bigvee_{A \in L^{X}}(\Phi(A) \odot A), B\right)=e_{L^{X}}(\sqcup \Phi, B) .
\end{aligned}
$$

(2) We have $\sqcap \Phi=\bigwedge_{A \in L^{X}}(\Phi(A) \rightarrow A)$ from:

$$
\begin{aligned}
& \bigwedge_{A \in L^{X}}\left(\Phi(A) \rightarrow e_{L^{X}}(B, A)=\bigwedge_{A \in L^{X}} e_{L^{X}}(B,(\Phi(A) \rightarrow A))\right. \\
& =e_{L^{X}}\left(B, \bigwedge_{A \in L^{X}}(\Phi(A) \rightarrow A)\right) .
\end{aligned}
$$

Definition 2.8. $[13,14]$ Let $\left(L^{X}, e_{L^{X}}\right)$ and $\left(L^{Y}, e_{L^{Y}}\right)$ be fuzzy posets. A operator $\mathcal{H}: L^{X} \rightarrow L^{Y}$ is a join preserving map if $\mathcal{H}(\sqcup \Phi)=\sqcup \mathcal{H}^{\rightarrow}(\Phi)$ for all $\Phi \in L^{L^{X}}$, where $\mathcal{H}^{\rightarrow}(\Phi)(B)=\bigvee_{\mathcal{H}(A)=B} \Phi(A)$. 
Theorem 2.9. [4] Let $X$ and $Y$ be two sets. Let $\left(L^{X}, e_{L^{X}}\right)$ and $\left(L^{Y}, e_{L^{Y}}\right)$ be fuzzy posets. Then $\mathcal{H}: L^{X} \rightarrow L^{Y}$ is a join preserving map iff $\mathcal{H}(\alpha \odot A)=$ $\alpha \odot \mathcal{H}(A)$ and $\mathcal{H}\left(\bigvee_{i \in I} A_{i}\right)=\bigvee_{i \in I} \mathcal{H}\left(A_{i}\right)$ for all $A, A_{i} \in L^{X}$, and $\alpha \in L$.

In this paper, $\mathcal{H}: L^{X} \rightarrow L^{Y}$ is called a join preserving map if $\mathcal{H}(\alpha \odot A)=$ $\alpha \odot \mathcal{H}(A)$ and $\mathcal{H}\left(\bigvee_{i \in I} A_{i}\right)=\bigvee_{i \in I} \mathcal{H}\left(A_{i}\right)$ for all $A, A_{i} \in L^{X}$, and $\alpha \in L$.

Lemma 2.10. $[1,2]$ Let $\left(L, \vee, \wedge, \odot, \rightarrow,{ }^{*}, \perp, \top\right)$ be a complete residuated lattice with a strong negation ${ }^{*}$. For each $x, y, z, x_{i}, y_{i} \in L$, the following properties hold.

(1) If $y \leq z$, then $x \odot y \leq x \odot z$.

(2) If $y \leq z$, then $x \rightarrow y \leq x \rightarrow z$ and $z \rightarrow x \leq y \rightarrow x$.

(3) $x \rightarrow y=\top$ iff $x \leq y$.

(4) $x \rightarrow \top=\top$ and $\top \rightarrow x=x$.

(5) $x \odot y \leq x \wedge y$.

(6) $x \odot\left(\bigvee_{i \in \Gamma} y_{i}\right)=\bigvee_{i \in \Gamma}\left(x \odot y_{i}\right)$ and $\left(\bigvee_{i \in \Gamma} x_{i}\right) \odot y=\bigvee_{i \in \Gamma}\left(x_{i} \odot y\right)$.

(7) $x \rightarrow\left(\bigwedge_{i \in \Gamma} y_{i}\right)=\bigwedge_{i \in \Gamma}\left(x \rightarrow y_{i}\right)$ and $\left(\bigvee_{i \in \Gamma} x_{i}\right) \rightarrow y=\bigwedge_{i \in \Gamma}\left(x_{i} \rightarrow y\right)$.

(8) $\bigvee_{i \in \Gamma} x_{i} \rightarrow \bigvee_{i \in \Gamma} y_{i} \geq \bigwedge_{i \in \Gamma}\left(x_{i} \rightarrow y_{i}\right)$ and $\bigwedge_{i \in \Gamma} x_{i} \rightarrow \bigwedge_{i \in \Gamma} y_{i} \geq \bigwedge_{i \in \Gamma}\left(x_{i} \rightarrow\right.$ $\left.y_{i}\right)$.

(9) $(x \rightarrow y) \odot x \leq y$ and $(y \rightarrow z) \odot(x \rightarrow y) \leq(x \rightarrow z)$.

(10) $x \rightarrow y \leq(y \rightarrow z) \rightarrow(x \rightarrow z)$ and $x \rightarrow y \leq(z \rightarrow x) \rightarrow(z \rightarrow y)$.

(11) $\bigwedge_{i \in \Gamma} x_{i}^{*}=\left(\bigvee_{i \in \Gamma} x_{i}\right)^{*}$ and $\bigvee_{i \in \Gamma} x_{i}^{*}=\left(\bigwedge_{i \in \Gamma} x_{i}\right)^{*}$.

(12) $(x \odot y) \rightarrow z=x \rightarrow(y \rightarrow z)=y \rightarrow(x \rightarrow z)$ and $(x \odot y)^{*}=x \rightarrow y^{*}$.

(13) $x^{*} \rightarrow y^{*}=y \rightarrow x$ and $(x \rightarrow y)^{*}=x \odot y^{*}$.

(14) $y \rightarrow z \leq x \odot y \rightarrow x \odot z$.

\section{Join Preserving Maps, Fuzzy Preorders and Alexandrov Fuzzy Topologies}

Definition 3.1. [4] A join preserving operator $\mathcal{H}: L^{X} \rightarrow L^{X}$ is called an upper approximation operator iff it satisfies the following conditions:

(H1) $A \leq \mathcal{H}(A)$,

(H2) $\mathcal{H}(\mathcal{H}(A)) \leq \mathcal{H}(A)$, for all $A \in L^{X}$.

Example 3.2. Let $R \in L^{X \times X}$ be a fuzzy relation. Define $\mathcal{H}_{R}: L^{X} \rightarrow L^{X}$ as follows

$$
\mathcal{H}_{R}(A)(y)=\bigvee_{x \in X}(A(x) \odot R(x, y)) .
$$


(1) Since $\mathcal{H}_{R}(\alpha \odot A)=\alpha \odot \mathcal{H}_{R}(A)$ and $\mathcal{H}_{R}\left(\bigvee_{i \in \Gamma} A_{i}\right)=\bigvee_{i \in \Gamma} \mathcal{H}_{R}\left(A_{i}\right)$, by Theorem $2.9, \mathcal{H}_{R}$ is a join preserving map.

(2) If $R$ is a fuzzy preorder, then $\mathcal{H}_{R}$ is an upper approximation operator from the followings:

$$
\begin{aligned}
& \mathcal{H}_{R}\left(\mathcal{H}_{R}(A)\right)(z)=\bigvee_{y \in X}\left(\mathcal{H}_{R}(A)(y) \odot R(y, z)\right) \\
& =\bigvee_{y \in X}\left(\left(\bigvee_{x \in X}(A(x) \odot R(x, y))\right) \odot R(y, z)\right) \\
& \leq \bigvee_{x \in X}(A(x) \odot R(x, z))=\mathcal{H}_{R}(A)(z)
\end{aligned}
$$

Theorem 3.3. Let $\mathcal{H}$ be an upper approximation operator. If $\mathcal{H}^{-1}$ is a join preserving map such that $\mathcal{H}^{-1}\left(\top_{x}\right)(y)=\mathcal{H}\left(\top_{y}\right)(x)$ for all $x, y \in X$, then $\mathcal{H}^{-1}$ is an upper approximation operator.

Proof. Since $A=\bigvee_{x \in X}\left(A(x) \odot \top_{x}\right)$, we have

(H1) Since $\mathcal{H}^{-1}\left(\top_{x}\right)(x)=\mathcal{H}\left(\top_{x}\right)(x) \geq \top_{x}(x)=\top$, we have

$$
\begin{aligned}
& \mathcal{H}^{-1}(A)(x)=\mathcal{H}^{-1}\left(\bigvee_{p \in X}\left(A(p) \odot \top_{p}\right)\right)(x) \\
& =\bigvee_{p \in X}\left(A(p) \odot \mathcal{H}^{-1}\left(\top_{p}\right)(x)\right) \\
& \geq A(x) \odot \mathcal{H}^{-1}\left(\top_{x}\right)(x)=A(x) .
\end{aligned}
$$

(H2)

$$
\begin{aligned}
& \mathcal{H}^{-1}\left(\mathcal{H}^{-1}(A)\right)(z)=\mathcal{H}^{-1}\left(\mathcal{H}^{-1}\left(\bigvee_{x \in X}\left(A(x) \odot \top_{x}\right)\right)(z)\right. \\
& =\bigvee_{x \in X}\left(A(x) \odot \mathcal{H}^{-1}\left(\mathcal{H}^{-1}\left(\top_{x}\right)\right)(z)\right. \\
& =\bigvee_{x \in X}\left(A(x) \odot \bigvee_{y \in X}\left(\mathcal{H}^{-1}\left(\top_{x}\right)(y) \odot \mathcal{H}^{-1}\left(\top_{y}\right)(z)\right)\right. \\
& =\bigvee_{x \in X}\left(A(x) \odot \bigvee_{y \in X}\left(\mathcal{H}\left(\top_{y}\right)(x) \odot \mathcal{H}\left(\top_{z}\right)(y)\right)\right. \\
& =\bigvee_{x \in X}\left(A(x) \odot \mathcal{H}\left(\top_{z}\right)(x)\right)=\bigvee_{x \in X}\left(A(x) \odot \mathcal{H}^{-1}\left(\top_{x}\right)(z)\right) \\
& =\mathcal{H}^{-1}(A)(z) .
\end{aligned}
$$

Theorem 3.4. Let $\mathcal{H}$ and $\mathcal{H}^{-1}$ be join preserving maps such that $\mathcal{H}^{-1}\left(\top_{x}\right)(y)$ $=\mathcal{H}\left(\top_{y}\right)(x)$ for all $x, y \in X$. Then we have the following properties.

$(1) \bigwedge_{A \in L^{X}}(\mathcal{H}(A)(x) \rightarrow \mathcal{H}(A)(z))=\bigwedge_{y \in X}\left(\mathcal{H}\left(\top_{y}\right)(x) \rightarrow \mathcal{H}\left(\top_{y}\right)(z)\right)$ and $\bigwedge_{A \in L^{X}}\left(\mathcal{H}^{-1}(A)(x) \rightarrow \mathcal{H}^{-1}(A)(z)\right)=\bigwedge_{y \in X}\left(\mathcal{H}^{-1}\left(\top_{y}\right)(x) \rightarrow \mathcal{H}^{-1}\left(\top_{y}\right)(z)\right)=$ $\bigwedge_{y \in X}\left(\mathcal{H}\left(\top_{x}\right)(y) \rightarrow \mathcal{H}\left(\top_{z}\right)(y)\right)$.

(2) If $\mathcal{H}$ and $\mathcal{H}^{-1}$ are upper approximation operators, then

$$
\begin{aligned}
\mathcal{H}\left(\top_{x}\right)(z) & =\bigwedge_{A \in L^{X}}(\mathcal{H}(A)(x) \rightarrow \mathcal{H}(A)(z)) \\
& =\bigwedge_{y \in X}\left(\mathcal{H}\left(\top_{y}\right)(x) \rightarrow \mathcal{H}\left(\top_{y}\right)(z)\right),
\end{aligned}
$$




$$
\begin{aligned}
\mathcal{H}^{-1}\left(\top_{z}\right)(x) & =\bigwedge_{A \in L^{X}}\left(\mathcal{H}^{-1}(A)(z) \rightarrow \mathcal{H}^{-1}(A)(x)\right) \\
& =\bigwedge_{y \in X}\left(\mathcal{H}^{-1}\left(\top_{y}\right)(z) \rightarrow \mathcal{H}^{-1}\left(\top_{y}\right)(x)\right) \\
& =\bigwedge_{y \in X}\left(\mathcal{H}\left(\top_{z}\right)(y) \rightarrow \mathcal{H}\left(\top_{x}\right)(y)\right) .
\end{aligned}
$$

Proof (1) Since $A=\bigvee_{x \in X}\left(A(x) \odot \top_{x}\right)$, we have

$$
\begin{aligned}
& \mathcal{H}(A)(x) \rightarrow \mathcal{H}(A)(z) \\
& =\mathcal{H}\left(\bigvee_{p \in X}\left(A(p) \odot \top_{p}\right)\right)(x) \rightarrow \mathcal{H}\left(\bigvee_{w \in X}\left(A(w) \odot \top_{w}\right)\right)(z) \\
& =\bigvee_{u \in X}\left(A(u) \odot \mathcal{H}\left(\top_{u}\right)(x)\right) \rightarrow \bigvee_{u \in X}\left(A(u) \odot \mathcal{H}\left(\top_{u}\right)(z)\right) \\
& \geq \bigwedge_{u \in X}\left(\left(A(u) \odot \mathcal{H}\left(\top_{u}\right)(x)\right) \rightarrow\left(A(u) \odot \mathcal{H}\left(\top_{u}\right)(z)\right)\right)(\text { Lemma } 2.10(8)) \\
& \left.\geq \bigwedge_{u \in X}\left(\mathcal{H}\left(\top_{u}\right)(x)\right) \rightarrow \mathcal{H}\left(\top_{u}\right)(z)\right) .(\text { Lemma } 2.10(14))
\end{aligned}
$$

(2) Since $\bigvee_{y \in X}\left(\mathcal{H}\left(\top_{x}\right)(y) \odot \mathcal{H}\left(\top_{y}\right)(z)\right)=\mathcal{H}\left(\bigvee_{y \in X}\left(\mathcal{H}\left(\top_{x}\right)(y) \odot \top_{y}\right)(z)\right)=$ $\mathcal{H}\left(\mathcal{H}\left(\top_{x}\right)\right)(z) \geq \mathcal{H}\left(\top_{x}\right)(z)$, then $\mathcal{H}\left(\top_{y}\right)(z) \leq \bigwedge_{x \in X}\left(\mathcal{H}\left(\top_{x}\right)(y) \rightarrow \mathcal{H}\left(\top_{x}\right)(z)\right) \leq$ $\mathcal{H}\left(\top_{y}\right)(y) \rightarrow \mathcal{H}\left(\top_{y}\right)(z) \leq \top_{y}(y) \rightarrow \mathcal{H}\left(\top_{y}\right)(z)=\mathcal{H}\left(\top_{y}\right)(z)$. By $(1), \mathcal{H}\left(\top_{y}\right)(z)=$ $\bigwedge_{x \in X}\left(\mathcal{H}\left(\top_{x}\right)(y) \rightarrow \mathcal{H}\left(\top_{x}\right)(z)\right)=\bigwedge_{A \in L^{X}}(\mathcal{H}(A)(x) \rightarrow \mathcal{H}(A)(z))$. Other case is similarly proved.

Example 3.5. Let $R \in L^{X \times X}$ be a fuzzy relation. Define $\mathcal{H}_{R}: L^{X} \rightarrow L^{X}$ as follows

$$
\begin{gathered}
\mathcal{H}_{R}(A)(y)=\bigvee_{x \in X}(A(x) \odot R(x, y)) \\
\mathcal{H}_{R}^{-1}(A)(y)=\mathcal{H}_{R^{-1}}(A)(y)=\bigvee_{x \in X}\left(A(x) \odot R^{-1}(x, y)\right)=\bigvee_{x \in X}(A(x) \odot R(y, x)) .
\end{gathered}
$$

(1) Since $\mathcal{H}_{R}\left(\top_{x}\right)(y)=R(x, y)$, for $R^{x}(y)=R(x, y)=R_{y}(x)$,

$$
\begin{aligned}
\bigwedge_{A \in L^{X}}\left(\mathcal{H}_{R}(A)(x) \rightarrow \mathcal{H}_{R}(A)(z)\right) & =\bigwedge_{y \in X}(R(y, x) \rightarrow R(y, z)) \\
& =\bigwedge_{y \in X}\left(R_{x}(y) \rightarrow R_{z}(y)\right) \\
\bigwedge_{A \in L^{X}}\left(\mathcal{H}_{R}^{-1}(A)(x) \rightarrow \mathcal{H}_{R}^{-1}(A)(z)\right) & =\bigwedge_{y \in X}(R(x, y) \rightarrow R(z, y)) \\
& =\bigwedge_{y \in X}\left(R^{x}(y) \rightarrow R^{z}(y)\right) .
\end{aligned}
$$

(2) If $R$ is a fuzzy preorder, then $R^{-1}$ is a fuzzy preorder. Moreover, $\mathcal{H}_{R}$ and $\mathcal{H}_{R}^{-1}$ are upper approximation operator such that

$$
\begin{aligned}
R(x, z) & =\mathcal{H}_{R}\left(\top_{x}\right)(z)=\bigwedge_{A \in L^{X}}\left(\mathcal{H}_{R}(A)(x) \rightarrow \mathcal{H}_{R}(A)(z)\right) \\
& =\bigwedge_{y \in X}(R(y, x) \rightarrow R(y, z))=\bigwedge_{y \in X}\left(R_{x}(y) \rightarrow R_{z}(y)\right) \\
& =\mathcal{H}_{R}^{-1}\left(\top_{z}\right)(x)=\bigwedge_{A \in L^{X}}\left(\mathcal{H}_{R}^{-1}(A)(z) \rightarrow \mathcal{H}_{R}^{-1}(A)(x)\right) \\
& =\bigwedge_{y \in X}(R(z, y) \rightarrow R(x, y))=\bigwedge_{y \in X}\left(R^{x}(y) \rightarrow R^{z}(y)\right)
\end{aligned}
$$


Definition 3.6. An operator $\mathbf{T}: L^{X} \rightarrow L$ is called an Alexandrov fuzzy topology on $X$ iff it satisfies the following conditions:

(T1) $\mathbf{T}(\alpha)=\top$,

(T2) $\mathbf{T}\left(\bigwedge_{i \in \Gamma} A_{i}\right) \geq \bigwedge_{i \in \Gamma} \mathbf{T}\left(A_{i}\right)$ and $\mathbf{T}\left(\bigvee_{i \in \Gamma} A_{i}\right) \geq \bigwedge_{i \in \Gamma} \mathbf{T}\left(A_{i}\right)$,

(T3) $\mathbf{T}(\alpha \odot A) \geq \mathbf{T}(A)$,

(T4) $\mathbf{T}(\alpha \rightarrow A) \geq \mathbf{T}(A)$.

Theorem 3.7. Let $\mathbf{T}: L^{X} \rightarrow L$ be an Alexandrov fuzzy topology. Define $\mathbf{T}^{*}(A)=\mathbf{T}\left(A^{*}\right)$. Then $\mathbf{T}^{*}$ is an Alexandrov fuzzy topology.

Proof. $(\mathrm{T} 1) \mathbf{T}^{*}(\alpha)=\mathbf{T}\left(\left(\alpha^{*}\right)=\top\right.$.

(T2) $\mathbf{T}^{*}\left(\bigvee_{i \in \Gamma} A_{i}\right)=\mathbf{T}\left(\bigwedge_{i \in \Gamma} A_{i}^{*}\right) \geq \bigwedge_{i \in \Gamma} \mathbf{T}\left(A_{i}^{*}\right)=\bigwedge_{i \in \Gamma} \mathbf{T}^{*}\left(A_{i}\right)$ and

$$
\mathbf{T}^{*}\left(\bigwedge_{i \in \Gamma} A_{i}\right)=\mathbf{T}\left(\bigvee_{i \in \Gamma} A_{i}^{*}\right) \geq \bigwedge_{i \in \Gamma} \mathbf{T}\left(A_{i}^{*}\right)=\bigwedge_{i \in \Gamma} \mathbf{T}^{*}\left(A_{i}\right) .
$$

$$
\begin{aligned}
\mathbf{T}^{*}(\alpha \odot A) & =\mathbf{T}\left((\alpha \odot A)^{*}\right)=\mathbf{T}\left(\alpha \rightarrow A^{*}\right) \\
& \geq \mathbf{T}\left(A^{*}\right)=\mathbf{T}^{*}(A) .
\end{aligned}
$$

$$
\begin{aligned}
\mathbf{T}^{*}(\alpha \rightarrow A) & =\mathbf{T}\left((\alpha \rightarrow A)^{*}\right)=\mathbf{T}\left(\left(\alpha \odot A^{*}\right)^{* *}\right) \\
& =\mathbf{T}\left(\alpha \odot A^{*}\right) \geq \mathbf{T}\left(A^{*}\right)=\mathbf{T}^{*}(A) .
\end{aligned}
$$

Theorem 3.8. Let $\mathcal{H}$ be a join preserving map. Define $\mathbf{T}_{H}: L^{X} \rightarrow L$ as

$$
\mathbf{T}_{H}(A)=\bigwedge_{x \in X}(\mathcal{H}(A)(x) \rightarrow A(x))=e_{L^{X}}(\mathcal{H}(A), A) .
$$

Then we have the following properties.

(1) $\mathbf{T}_{H}$ is an Alexandrov fuzzy topology on $X$.

(2) $\mathbf{T}_{H}(A)=\bigwedge_{x, y \in X}\left(\mathcal{H}\left(\top_{x}\right)(y) \rightarrow(A(x) \rightarrow A(y))\right.$ such that $\mathbf{T}_{H}(A) \geq$ $\bigwedge_{x \neq y \in X} \mathcal{H}^{*}\left(\top_{x}\right)(y)$.

(3) If $\mathcal{H}$ is an upper approximation operator, then $\mathbf{T}_{H}\left(\mathcal{H}\left(\top_{x}\right)\right)=\top$.

(4) If $\mathcal{H}^{-1}$ is a join preserving map such that $\mathcal{H}^{-1}\left(\top_{x}\right)(y)=\mathcal{H}\left(\top_{y}\right)(x)$ for all $x, y \in X$. Define $\mathbf{T}_{H}^{*}(A)=\mathbf{T}_{H}\left(A^{*}\right)$. Then $\mathbf{T}_{H}^{*}=\mathbf{T}_{H^{-1}}$ is an Alexandrov fuzzy topology.

(5) If $\mathcal{H}$ is an upper approximation operator, then $\mathcal{H}^{-1}$ is an upper approximation operator such that

$$
\mathbf{T}_{H}\left(\mathcal{H}^{-1 *}\left(\top_{x}\right)\right)=\mathbf{T}_{H^{-1}}\left(\mathcal{H}^{*}\left(\top_{x}\right)\right)=\top .
$$


Proof. (1) For $\alpha=\bigvee_{y \in X}\left(\alpha(y) \odot \top_{y}\right), \mathcal{H}(\alpha)(x)=\mathcal{H}\left(\bigvee_{y \in X}\left(\alpha(y) \odot \top_{y}\right)\right)(x)=$ $\bigvee_{y \in X}\left(\alpha(y) \odot \mathcal{H}\left(\top_{y}\right)(x)\right)=\alpha \odot \bigvee_{y \in X} \mathcal{H}\left(\top_{y}\right)(x) \leq \alpha$. Thus,

$$
\left(\mathbf{T}_{H}\right)(\alpha)=\bigwedge_{x}(\mathcal{H}(\alpha)(x) \rightarrow \alpha(x))=\top .
$$

$$
\begin{aligned}
\mathbf{T}_{H}\left(\bigvee_{i \in \Gamma} A_{i}\right) & =\bigwedge_{x}\left(\mathcal{H}\left(\bigvee_{i \in \Gamma} A_{i}\right)(x) \rightarrow \bigvee_{i \in \Gamma} A_{i}(x)\right) \\
& =\bigwedge_{x}\left(\bigvee_{i \in \Gamma} \mathcal{H}\left(A_{i}\right)(x) \rightarrow \bigvee_{i \in \Gamma} A_{i}(x)\right) \\
& \geq \bigwedge_{x}\left(\bigwedge_{i \in \Gamma}\left(\mathcal{H}\left(A_{i}\right)(x) \rightarrow A_{i}(x)\right)\right)(\text { by Lemma 2.10(8)) } \\
& =\bigwedge_{i \in \Gamma} \mathbf{T}_{H}\left(A_{i}\right)
\end{aligned}
$$

Since $\mathcal{H}\left(\bigwedge_{i \in \Gamma} A_{i}\right) \leq \bigwedge_{i \in \Gamma} \mathcal{H}\left(A_{i}\right)$, we have

$$
\begin{aligned}
\mathbf{T}_{H}\left(\bigwedge_{i \in \Gamma} A_{i}\right) & =\bigwedge_{x}\left(\mathcal{H}\left(\bigwedge_{i \in \Gamma} A_{i}\right)(x) \rightarrow \bigwedge_{i \in \Gamma} A_{i}(x)\right) \\
& \geq \bigwedge_{x}\left(\bigwedge_{i \in \Gamma} \mathcal{H}\left(A_{i}\right)(x) \rightarrow \bigwedge_{i \in \Gamma} A_{i}(x)\right) \\
& \geq \bigwedge_{x}\left(\bigwedge_{i \in \Gamma}\left(\mathcal{H}\left(A_{i}\right)(x) \rightarrow A_{i}(x)\right)\right)(\text { by Lemma 2.10(8)) } \\
& =\bigwedge_{i \in \Gamma} \mathbf{T}_{H}\left(A_{i}\right) .
\end{aligned}
$$

Since

$$
\begin{aligned}
\alpha \odot A(x) & \odot(A(x) \rightarrow A(y)) \leq \alpha \odot A(y) \\
\text { iff } A(x) & \rightarrow A(y) \leq \alpha \odot A(x) \rightarrow \alpha \odot A(y) \\
\mathbf{T}_{H}(\alpha \odot A) & =\bigwedge_{x}(\mathcal{H}(\alpha \odot A)(x) \rightarrow \alpha \odot A(x)) \\
& =\bigwedge_{x}(\alpha \odot \mathcal{H}(A)(x) \rightarrow \alpha \odot A(x)) \\
& \geq \bigwedge_{x}(\mathcal{H}(A)(x) \rightarrow A(x))=\mathbf{T}_{H}(A) .
\end{aligned}
$$

Since

$$
\begin{aligned}
& \alpha \odot(\alpha \rightarrow A(x)) \odot(A(x) \rightarrow A(y)) \leq A(x) \odot(A(x) \rightarrow A(y)) \leq A(y) \\
& \text { iff } A(x) \rightarrow A(y) \leq(\alpha \rightarrow A(x)) \rightarrow(\alpha \rightarrow A(y)) \\
& \alpha \odot \mathcal{H}(\alpha \rightarrow A)=\mathcal{H}(\alpha \odot(\alpha \rightarrow A)) \leq \mathcal{H}(A) \\
& \text { iff } \mathcal{H}(\alpha \rightarrow A) \leq \alpha \rightarrow \mathcal{H}(A) \\
& \mathbf{T}_{H}(\alpha \rightarrow A) \quad \bigwedge_{x}(\mathcal{H}(\alpha \rightarrow A)(x) \rightarrow(\alpha \rightarrow A(x))) \\
& \geq \bigwedge_{x}((\alpha \rightarrow \mathcal{H}(A)(x)) \rightarrow(\alpha \rightarrow A(x))) \\
& \geq \bigwedge_{x}(\mathcal{H}(A)(x) \rightarrow A(x))=\mathbf{T}_{H}(A) . \text { (by Lemma 2.10(10)) }
\end{aligned}
$$

(2) For $A=\bigvee_{x \in X}\left(A(x) \odot \top_{x}\right)$, we have

$$
\begin{aligned}
\mathbf{T}_{H}(A) & =\bigwedge_{y \in X}(\mathcal{H}(A)(y) \rightarrow A(y)) \\
& =\bigwedge_{y \in X}\left(\mathcal{H}\left(\bigvee_{x \in X}\left(A(x) \odot \top_{x}\right)\right)(y) \rightarrow A(y)\right) \\
& =\bigwedge_{y \in X}\left(\bigvee_{x \in X}\left(A(x) \odot \mathcal{H}\left(\top_{x}\right)(y) \rightarrow A(y)\right)\right. \\
& =\bigwedge_{x, y \in X}\left(\mathcal{H}\left(\top_{x}\right)(y) \rightarrow(A(x) \rightarrow A(y))\right.
\end{aligned}
$$




$$
\begin{aligned}
\mathbf{T}_{H}(A) & =\bigwedge_{y \in X}\left(\bigvee_{x \in X}\left(A(x) \odot \mathcal{H}\left(\top_{x}\right)(y) \rightarrow A(y)\right)\right. \\
& =\bigwedge_{y \in X}\left(\bigvee_{x \in X}\left(A(x) \odot \mathcal{H}\left(\top_{x}\right)(y) \rightarrow \bigvee_{x \in X}\left(A(x) \odot \top_{x}(y)\right)\right)\right. \\
& \geq \bigwedge_{x, y \in X}\left(\mathcal{H}\left(\top_{x}\right)(y) \rightarrow \top_{x}(y)\right)=\bigwedge_{x \neq y \in X} \mathcal{H}^{*}\left(\top_{x}\right)(y)
\end{aligned}
$$

(3)

$$
\begin{aligned}
\mathbf{T}_{H}\left(\mathcal{H}\left(\top_{z}\right)\right) & =\bigwedge_{x, y \in X}\left(\mathcal{H}\left(\top_{x}\right)(y) \rightarrow\left(\mathcal{H}\left(\top_{z}\right)(x) \rightarrow \mathcal{H}\left(\top_{z}\right)(y)\right)\right. \\
& =\bigwedge_{y \in X}\left(\bigvee_{x \in X}\left(\mathcal{H}\left(\top_{x}\right)(y) \odot \mathcal{H}\left(\top_{z}\right)(x)\right) \rightarrow \mathcal{H}\left(\top_{z}\right)(y)\right) \\
& =\bigwedge_{y \in X}\left(\mathcal{H}\left(\bigvee_{x \in X}\left(\mathcal{H}\left(\top_{z}\right)(x) \odot \top_{x}\right)(y) \rightarrow \mathcal{H}\left(\top_{z}\right)(y)\right)\right. \\
& =\bigwedge_{y \in X}\left(\left(\mathcal{H}\left(\mathcal{H}\left(\top_{z}\right)\right)(y) \rightarrow \mathcal{H}\left(\top_{z}\right)(y)\right)\right. \\
& \left.=\bigwedge_{y \in X}\left(\mathcal{H}\left(\top_{z}\right)\right)(y) \rightarrow \mathcal{H}\left(\top_{z}\right)(y)\right)=\top
\end{aligned}
$$

(4) By (2),

$$
\begin{aligned}
\mathbf{T}_{H^{-1}}(A) & =\bigwedge_{x, y \in X}\left(\mathcal{H}^{-1}\left(\top_{x}\right)(y) \rightarrow(A(x) \rightarrow A(y))\right. \\
& =\bigwedge_{x, y \in X}\left(\mathcal{H}\left(\top_{y}\right)(x) \rightarrow\left(A^{*}(y) \rightarrow A^{*}(y)\right)\right. \\
& =\mathbf{T}_{H}\left(A^{*}\right)=\mathbf{T}_{H}^{*}(A) .
\end{aligned}
$$

(5)

$$
\begin{aligned}
& \mathbf{T}_{H}\left(\mathcal{H}^{-1 *}\left(\top_{x}\right)\right)=\mathbf{T}_{H^{-1}}\left(\mathcal{H}^{-1}\left(\top_{x}\right)\right)=\top \\
& \mathbf{T}_{H^{-1}}\left(\mathcal{H}^{*}\left(\top_{x}\right)\right)=\mathbf{T}_{H}\left(\mathcal{H}\left(\top_{x}\right)\right)=\top
\end{aligned}
$$

Example 3.9. Let $R \in L^{X \times X}$ be a fuzzy relation. By Example 2.2, $\mathcal{H}_{R}$ is a join preserving map with $\mathcal{H}_{R}(A)(y)=\bigvee_{x \in X}(A(x) \odot R(x, y))$. Define $\mathbf{T}_{H_{R}}: L^{X} \rightarrow L$ as

$$
\mathbf{T}_{H_{R}}(A)=\bigwedge_{x \in X}\left(\mathcal{H}_{R}(A)(x) \rightarrow A(x)\right)=e_{L^{X}}\left(\mathcal{H}_{R}(A), A\right) .
$$

From Theorem, we obtain the following results.

(1) $\mathbf{T}_{H_{R}}$ is an Alexandrov fuzzy topology on $X$.

(2) Since $\mathcal{H}_{R}\left(\top_{x}\right)(y)=R(x, y)$, we have $\mathbf{T}_{H_{R}}(A)=\bigwedge_{x, y \in X}(R(x, y) \rightarrow$ $(A(x) \rightarrow A(y)))$ such that $\mathbf{T}_{H_{R}}(A) \geq \bigwedge_{x \neq y \in X} R^{*}(x, y)$.

(3) If $R$ is a preorder, then $\mathcal{H}_{R}$ is an upper approximation operator such that $\mathbf{T}_{H_{R}}\left(R^{x}\right)=\top$ for all $x \in X$.

(4) If $R^{-1}(x, y)=R(x, y)$ for all $x, y \in X$, then $\mathcal{H}_{R^{-1}}$ is a join preserving map. Define $\mathbf{T}_{H_{R}}^{*}(A)=\mathbf{T}_{H_{R}}\left(A^{*}\right)$. Then $\mathbf{T}_{H_{R}}^{*}=\mathbf{T}_{H_{R^{-1}}}$ is an Alexandrov fuzzy topology. 
(5) If $R$ is a fuzzy preorder, then $R^{-1}$ is a fuzzy preorder. Since $\mathcal{H}_{R}^{-1 *}\left(\top_{x}\right)=$ $R_{x}^{*}$ and $\mathcal{H}_{R}^{*}\left(\top_{x}\right)=\left(R^{x}\right)^{*}$, then $\mathcal{H}_{R}$ and $\mathcal{H}_{R^{-1}}$ are upper approximation operators such that

$$
\mathbf{T}_{H_{R}}\left(R_{x}^{*}\right)=\mathbf{T}_{H_{R^{-1}}}\left(\left(R^{x}\right)^{*}\right)=\top
$$

Theorem 3.10. Let $\mathcal{H}, \mathcal{H}^{-1}: L^{X} \rightarrow L^{X}$ be join preserving maps. Then we have the following properties.

(1) $\mathbf{T}_{H}(A) \leq \mathbf{T}_{H^{-1}}(A \rightarrow \alpha)$ and $\mathbf{T}_{H^{-1}}(A) \leq \mathbf{T}_{H}(A \rightarrow \alpha)$.

(2) $\mathbf{T}_{H}(A) \leq \mathbf{T}_{H^{-1}}(A \rightarrow \alpha) \leq \mathbf{T}_{H^{-1}}((A \rightarrow \alpha) \rightarrow \beta)$ and $\mathbf{T}_{H^{-1}}(A)=$ $\mathbf{T}_{H}\left(A^{*}\right)$.

Proof. (1)

$$
\begin{aligned}
& A(x) \odot(A(x) \rightarrow A(y)) \odot(A(y) \rightarrow \alpha) \leq \alpha \\
& \text { iff }(A(x) \rightarrow A(y)) \odot(A(y) \rightarrow \alpha) \leq A(x) \rightarrow \alpha \\
& \text { iff }(A(x) \rightarrow A(y)) \leq(A(y) \rightarrow \alpha) \rightarrow(A(x) \rightarrow \alpha) \\
& \mathbf{T}_{H}(A)=\bigwedge_{x, y}\left(\mathcal{H}\left(\top_{x}\right)(y) \rightarrow(A(x) \rightarrow A(y))\right. \\
& \leq \bigwedge_{x, y}\left(\mathcal{H}\left(\top_{x}\right)(y) \rightarrow((A(y) \rightarrow \alpha) \rightarrow(A(x) \rightarrow \alpha))\right. \\
& \leq \bigwedge_{x, y}\left(\mathcal{H}^{-1}\left(\top_{y}\right)(x) \rightarrow((A(y) \rightarrow \alpha) \rightarrow(A(x) \rightarrow \alpha))\right. \\
&=\mathbf{T}_{H^{-1}}(A \rightarrow \alpha) .
\end{aligned}
$$

(2) By $(1), \mathbf{T}_{H}(A) \leq\left(\mathbf{T}_{H^{-1}}(A \rightarrow \alpha) \leq \mathbf{T}_{H}((A \rightarrow \alpha) \rightarrow \beta)\right.$. Put $\alpha=\beta=$ $\perp$. Then

$$
\mathbf{T}_{H}(A) \leq \mathbf{T}_{H^{-1}}\left(A^{*}\right) \leq \mathbf{T}_{H}\left(A^{* *}\right)=\mathbf{T}_{H}(A)
$$

Theorem 3.11. Let $\mathcal{H}, \mathcal{H}^{-1}: L^{X} \rightarrow L^{X}$ be join preserving maps.

(1) $\mathcal{H}\left(\top_{x}\right)(y) \leq \bigwedge_{A \in L^{X}}\left(\mathbf{T}_{H}(A) \rightarrow(A(x) \rightarrow A(y))\right.$. for all $x, y \in L^{X}$.

(2) $\mathcal{H}\left(\top_{x}\right)(y) \leq \bigwedge_{A \in L^{X}}\left(\mathbf{T}_{H^{-1}}(A) \rightarrow(A(y) \rightarrow A(x))\right.$. for all $x, y \in L^{X}$.

(3) If $\mathcal{H}$ is an upper approximation operator, then, for all $x, y \in L^{X}$,

$$
\begin{aligned}
\mathcal{H}\left(\top_{x}\right)(y) & =\bigwedge_{A \in L^{X}}\left(\mathbf{T}_{H}(A) \rightarrow(A(x) \rightarrow A(y))\right. \\
& =\bigwedge_{A \in L^{X}}\left(\mathbf{T}_{H^{-1}}(A) \rightarrow(A(y) \rightarrow A(x))\right. \\
& =\bigwedge_{z \in X}\left(\mathcal{H}\left(\top_{z}\right)(x) \rightarrow \mathcal{H}\left(\top_{z}\right)(y)\right) \\
& =\bigwedge_{z \in X}\left(\mathcal{H}^{*}\left(\top_{z}\right)(y) \rightarrow \mathcal{H}^{*}\left(\top_{z}\right)(x)\right) \\
& =\bigwedge_{z \in X}\left(\mathcal{H}^{-1}\left(\top_{z}\right)(y) \rightarrow \mathcal{H}^{-1}\left(\top_{z}\right)(x)\right) \\
& =\bigwedge_{z \in X}\left(\mathcal{H}^{-1 *}\left(\top_{z}\right)(x) \rightarrow \mathcal{H}^{-1 *}\left(\top_{z}\right)(y)\right)
\end{aligned}
$$


Proof. (1) Since $a \leq(a \rightarrow b) \rightarrow b$, we have

$$
\begin{aligned}
\mathcal{H}\left(\top_{x}\right)(y) & \leq \bigwedge_{A \in L^{X}}\left(\left(\mathcal{H}\left(\top_{x}\right)(y) \rightarrow(A(x) \rightarrow A(y)) \rightarrow(A(x) \rightarrow A(y))\right)\right. \\
& \leq \bigwedge_{A \in L^{X}}\left(\bigwedge_{s, t}\left(\left(\mathcal{H}\left(\top_{s}\right)(t) \rightarrow(A(s) \rightarrow A(t))\right) \rightarrow(A(x) \rightarrow A(y))\right)\right. \\
& =\bigwedge_{A \in L^{X}}\left(\mathbf{T}_{H}(A) \rightarrow(A(x) \rightarrow A(y))\right)
\end{aligned}
$$

$(2)$

$$
\begin{aligned}
& \mathcal{H}\left(\top_{x}\right)(y)=\mathcal{H}^{-1}\left(\top_{y}\right)(x) \leq \bigwedge_{A \in L^{X}}\left(\mathbf{T}_{H^{-1}}(A) \rightarrow(A(y) \rightarrow A(x))\right) \\
& \text { (3) Since } \mathbf{T}_{H}\left(\mathcal{H}\left(\top_{x}\right)\right)=\mathbf{T}_{H^{-1}}\left(\mathcal{H}^{-1}\left(\top_{x}\right)\right)=\mathbf{T}_{H}\left(\mathcal{H}^{-1 *}\left(\top_{x}\right)\right)=\mathbf{T}_{H^{-1}}\left(\mathcal{H}^{*}\left(\top_{x}\right)\right) \\
& =\top \text {, } \\
& \mathcal{H}\left(\top_{x}\right)(y) \leq \bigwedge_{A \in L^{X}}\left(\mathbf{T}_{H}(A) \rightarrow(A(x) \rightarrow A(y))\right) \\
& \leq \bigwedge_{z \in X}\left(\mathbf{T}_{H}\left(\mathcal{H}\left(\top_{z}\right)\right) \rightarrow\left(\mathcal{H}\left(\top_{z}\right)(x) \rightarrow \mathcal{H}\left(\top_{z}\right)(y)\right)\right) \\
& =\bigwedge_{z \in X}\left(\mathcal{H}\left(\top_{z}\right)(x) \rightarrow \mathcal{H}\left(\top_{z}\right)(y)\right) \leq \top_{x}(x) \rightarrow \mathcal{H}\left(\top_{x}\right)(y)=\mathcal{H}\left(\top_{x}\right)(y) . \\
& \mathcal{H}\left(\top_{x}\right)(y) \leq \bigwedge_{A \in L^{X}}\left(\mathbf{T}_{H}(A) \rightarrow(A(x) \rightarrow A(y))\right) \\
& \leq \bigwedge_{z \in X}\left(\mathbf{T}_{H}\left(\mathcal{H}^{-1 *}\left(\top_{z}\right)\right) \rightarrow\left(\mathcal{H}^{-1 *}\left(\top_{z}\right)(x) \rightarrow \mathcal{H}^{-1 *}\left(\top_{z}\right)(y)\right)\right) \\
& =\bigwedge_{z \in X}\left(\mathcal{H}^{-1}\left(\top_{z}\right)(y) \rightarrow \mathcal{H}^{-1}\left(\top_{z}\right)(x)\right) \\
& \begin{aligned}
& \leq \top_{y}(y) \rightarrow \mathcal{H}^{-1}\left(\top_{y}\right)(x)=\mathcal{H}\left(\top_{x}\right)(y) . \\
\mathcal{H}\left(\top_{x}\right)(y) & \leq \bigwedge_{A \in L_{X}}\left(\mathbf{T}_{H^{-1}}(A) \rightarrow(A(y) \rightarrow A(x))\right.
\end{aligned} \\
& \leq \bigwedge_{z \in X}\left(\mathbf{T}_{H^{-1}}\left(\mathcal{H}^{*}\left(\top_{z}\right)\right) \rightarrow\left(\mathcal{H}^{*}\left(\top_{z}\right)(y) \rightarrow \mathcal{H}^{*}\left(\top_{z}\right)(x)\right)\right. \\
& \leq \bigwedge_{z \in X}\left(\mathcal{H}^{*}\left(\top_{z}\right)(y) \rightarrow \mathcal{H}^{*}\left(\top_{z}\right)(x)\right) \\
& \left.\leq \mathcal{H}^{*}\left(\top_{x}\right)(y) \rightarrow \mathcal{H}^{*}\left(\top_{x}\right)(x)\right) \leq \mathcal{H}\left(\top_{x}\right)(y) \\
& \mathcal{H}\left(\top_{x}\right)(y) \leq \bigwedge_{A \in L^{X}}\left(\mathbf{T}_{H^{-1}}(A) \rightarrow(A(y) \rightarrow A(x))\right. \\
& \leq \bigwedge_{z \in X}\left(\mathbf{T}_{H^{-1}}\left(\mathcal{H}^{-1}\left(\top_{x}\right)\right) \rightarrow\left(\mathcal{H}^{-1}\left(\top_{z}\right)(y) \rightarrow \mathcal{H}^{-1}\left(\top_{z}\right)(x)\right)\right. \\
& \leq \bigwedge_{z \in X}\left(\mathcal{H}^{-1}\left(\top_{z}\right)(y) \rightarrow \mathcal{H}^{-1}\left(\top_{z}\right)(x)\right) \\
& \left.\leq \mathcal{H}\left(\top_{y}\right)(y) \rightarrow \mathcal{H}\left(\top_{x}\right)(y)\right) \leq \mathcal{H}\left(\top_{x}\right)(y) \text {. }
\end{aligned}
$$

Example 3.12. Let $R \in L^{X \times X}$ be a fuzzy relation and $\mathcal{H}_{R}, \mathcal{H}_{R^{-1}}$ be join preserving maps in Example 3.5.

(1) $R(x, y) \leq \bigwedge_{A \in L^{X}}\left(\mathbf{T}_{H_{R}}(A) \rightarrow(A(x) \rightarrow A(y))\right.$. for all $x, y \in L^{X}$.

(2) $R(x, y) \leq \bigwedge_{A \in L^{X}}\left(\mathbf{T}_{H_{R^{-1}}}(A) \rightarrow(A(y) \rightarrow A(x))\right.$. for all $x, y \in L^{X}$.

(3) If $R$ is a fuzzy preorder, then for all $x, y \in L^{X}$,

$$
\begin{aligned}
R(x, y) & =\bigwedge_{A \in L^{X}}\left(\mathbf{T}_{H_{R}}(A) \rightarrow(A(x) \rightarrow A(y))\right. \\
& =\bigwedge_{A \in L^{X}}\left(\mathbf{T}_{H_{R^{-1}}}(A) \rightarrow(A(y) \rightarrow A(x))\right. \\
& =\bigwedge_{z \in X}\left(R^{z}(x) \rightarrow R^{z}(y)\right) \\
& =\bigwedge_{z \in X}\left(\left(R^{z}\right)^{*}(y) \rightarrow\left(R^{z}\right)^{*}(x)\right) \\
& =\bigwedge_{z \in X}\left(R_{z}(y) \rightarrow R_{z}(x)\right) \\
& =\bigwedge_{z \in X}\left(R_{z}^{*}(x) \rightarrow R_{z}^{*}(y)\right) .
\end{aligned}
$$


Example 3.13. Let $\left(L=[0,1], \odot, \rightarrow{ }^{*}\right)$ be a complete residuated lattice with a strong negation which is defined by

$$
x \odot y=(x+y-1) \vee 0, x \rightarrow y=(1-x+y) \wedge 1, x^{*}=1-x .
$$

Let $X=\{x, y, z\}$ and $A, B \in L^{X}$ as follows:

$$
A(x)=0.9, A(y)=0.8, A(z)=0.3, \quad B(x)=0.3, B(y)=0.7, B(z)=0.8
$$

Let $\mathcal{H}, \mathcal{H}^{-1}: L^{X} \rightarrow L^{X}$ be a join preserving map such that, for all $x, y \in X$, $\mathcal{H}\left(1_{x}\right)(y)=\mathcal{H}^{-1}\left(1_{y}\right)(x)$ as follows

$$
\left(\begin{array}{ccc}
\mathcal{H}\left(1_{x}\right)(x)=0.5 & \mathcal{H}\left(1_{x}\right)(y)=0.2 & \mathcal{H}\left(1_{x}\right)(z)=1 \\
\mathcal{H}\left(1_{y}\right)(x)=0.8 & \mathcal{H}\left(1_{y}\right)(y)=0.6 & \mathcal{H}\left(1_{y}\right)(z)=0.9 \\
\mathcal{H}\left(1_{z}\right)(x)=1 & \mathcal{H}\left(1_{z}\right)(y)=0.5 & \mathcal{H}\left(1_{z}\right)(z)=0.7
\end{array}\right)
$$

(1) Since $1=\bigvee_{y \in X}\left(\mathcal{H}\left(1_{x}\right)(y) \odot \mathcal{H}\left(1_{y}\right)(x)=\mathcal{H}\left(\mathcal{H}\left(1_{x}\right)\right)(x) \neq \mathcal{H}\left(1_{x}\right)(x)=0.5\right.$ and $\mathcal{H}\left(1_{x}\right)(x)=0.5 \neq 1$, then $\mathcal{H}$ is not an upper approximation operator. Since $\mathcal{H}(A)(y)=\bigvee_{x \in X}\left(A(x) \odot\left(\mathcal{H}\left(1_{x}\right)(y)\right)\right.$, we have

$$
\mathcal{H}(A)=(0.6,0.4,0.9), \mathcal{H}(B)=(0.8,0.3,0.6) .
$$

Moreover, by Theorem 3.8,

$$
\begin{gathered}
\mathbf{T}_{H}(A)=e_{L^{X}}(\mathcal{H}(A), A)=0.4, \mathbf{T}_{H}(B)=e_{L^{X}}(\mathcal{H}(B), B)=0.5 . \\
\mathcal{H}\left(\top_{x}\right)=(0.5,0.2,1), \quad \mathcal{H}\left(\mathcal{H}\left(\top_{x}\right)\right)=(1,0.5,0.7) \\
\mathcal{H}\left(\top_{y}\right)=(0.8,0.6,0.9), \quad \mathcal{H}\left(\mathcal{H}\left(\top_{y}\right)\right)=(0.9,0.4,0.8) \\
\mathcal{H}\left(\top_{z}\right)=(1,0.5,0.7), \quad \mathcal{H}\left(\mathcal{H}\left(\top_{x}\right)\right)=(0.7,0.2,1) \\
\mathbf{T}_{H}\left(\mathcal{H}\left(\top_{x}\right)\right)=e_{L^{X}}\left(\mathcal{H}\left(\mathcal{H}\left(\top_{x}\right)\right), \mathcal{H}\left(\top_{x}\right)\right)=0.5 . \\
\mathbf{T}_{H}\left(\mathcal{H}\left(\top_{y}\right)\right)=e_{L^{X}}\left(\mathcal{H}\left(\mathcal{H}\left(\top_{y}\right)\right), \mathcal{H}\left(\top_{y}\right)\right)=0.8 . \\
\mathbf{T}_{H}\left(\mathcal{H}\left(\top_{z}\right)\right)=e_{L^{X}}\left(\mathcal{H}\left(\mathcal{H}\left(\top_{z}\right)\right), \mathcal{H}\left(\top_{z}\right)\right)=0.7 . \\
0.9=\mathcal{H}\left(\top_{y}\right)(z) \neq \bigwedge_{x \in X}\left(\mathbf{T}_{H}\left(\mathcal{H}\left(\top_{x}\right)\right) \rightarrow\left(\mathcal{H}\left(\top_{x}\right)(y) \rightarrow \mathcal{H}\left(\top_{x}\right)(z)\right)\right) \\
=(0.5 \rightarrow(0.2 \rightarrow 1)) \wedge(0.8 \rightarrow(0.6 \rightarrow 0.9)) \wedge(0.7 \rightarrow(0.5 \rightarrow 0.7)) \\
=1 \quad
\end{gathered}
$$

Hence, by Theorems $3.8(3)$ and $3.11(3)$, if $\mathcal{H}$ is not an upper approximation operator, the results does not hold.

(2) We obtain $\mathcal{H}^{-1}\left(1_{x}\right)(y)=\mathcal{H}\left(1_{y}\right)(x)$ as follows

$$
\left(\begin{array}{ccc}
\mathcal{H}^{-1}\left(1_{x}\right)(x)=0.5 & \mathcal{H}^{-1}\left(1_{x}\right)(y)=0.8 & \mathcal{H}^{-1}\left(1_{x}\right)(z)=1 \\
\mathcal{H}^{-1}\left(1_{y}\right)(x)=0.2 & \mathcal{H}^{-1}\left(1_{y}\right)(y)=0.6 & \mathcal{H}^{-1}\left(1_{y}\right)(z)=0.5 \\
\mathcal{H}^{-1}\left(1_{z}\right)(x)=1 & \mathcal{H}^{-1}\left(1_{z}\right)(y)=0.9 & \mathcal{H}^{-1}\left(1_{z}\right)(z)=0.7
\end{array}\right)
$$


Since $1=\bigvee_{y \in X}\left(\mathcal{H}^{-1}\left(1_{x}\right)(y) \odot \mathcal{H}^{-1}\left(1_{y}\right)(x)=\mathcal{H}^{-1}\left(\mathcal{H}^{-1}\left(1_{x}\right)(x) \neq \mathcal{H}^{-1}\left(1_{x}\right)(x)=\right.\right.$ 0.5 , then $\mathcal{H}^{-1}$ is not an upper approximation operator. Since $\mathcal{H}^{-1}(A)(y)=$ $\bigvee_{x \in X}\left(A(x) \odot\left(\mathcal{H}^{-1}\left(1_{x}\right)(y)\right)\right.$, we have

$$
\mathcal{H}^{-1}(A)=(0.4,0.7,0.9), \mathcal{H}^{-1}(B)=(0.8,0.7,0.5) .
$$

Moreover, by Theorem 3.8,

$$
\mathbf{T}_{H^{-1}}(A)=e_{L^{X}}\left(\mathcal{H}^{-1}(A), A\right)=0.4, \mathbf{T}_{H^{-1}}(B)=e_{L^{X}}\left(\mathcal{H}^{-1}(B), B\right)=0.5 .
$$

For $A^{*}=(0.1,0.2,0.7), \mathcal{H}^{-1}\left(A^{*}\right)=(0.7,0.6,0.4), \mathcal{H}\left(A^{*}\right)=(0.7,0.2,0.4)$ and

$$
\begin{gathered}
\mathbf{T}_{H^{-1}}(A)=\mathbf{T}_{H}\left(A^{*}\right)=e_{L^{X}}\left(\mathcal{H}\left(A^{*}\right), A^{*}\right)=0.4 \\
\mathbf{T}_{H}(A)=\mathbf{T}_{H^{-1}}\left(A^{*}\right)=e_{L^{X}}\left(\mathcal{H}^{-1}\left(A^{*}\right), A^{*}\right)=0.4 . \\
\mathcal{H}\left(\mathcal{H}^{-1 *}\left(1_{x}\right)\right)=(0,0,0.5) \neq \mathcal{H}^{-1 *}\left(1_{x}\right)=(0.5,0.2,0) \\
\mathcal{H}\left(\mathcal{H}^{-1 *}\left(1_{y}\right)\right)=(0.5,0,0.8) \neq \mathcal{H}^{-1 *}\left(1_{y}\right)=(0.8,0.4,0.5) \\
\mathcal{H}\left(\mathcal{H}^{-1 *}\left(1_{z}\right)\right)=(0.3,0,0) \neq \mathcal{H}^{-1}\left(1_{z}\right)=(0,0.1,0.3) \\
\left.\mathbf{T}_{H}\left(\mathcal{H}^{-1 *}\left(1_{x}\right)\right)=e_{L^{X}}\left(\left(\mathcal{H} \mathcal{H}^{-1 *}\left(1_{x}\right)\right), \mathcal{H}^{-1 *}\left(1_{x}\right)\right)\right)=0.5 \\
\left.\mathbf{T}_{H}\left(\mathcal{H}^{-1 *}\left(1_{y}\right)\right)=e_{L^{X}}\left(\mathcal{H}\left(\mathcal{H}^{-1 *}\left(1_{y}\right)\right), \mathcal{H}^{-1 *}\left(1_{y}\right)\right)\right)=0.7 \\
\left.\mathbf{T}_{H}\left(\mathcal{H}^{-1 *}\left(1_{z}\right)\right)=e_{L^{X}}\left(\mathcal{H}\left(\mathcal{H}^{-1 *}\left(1_{z}\right)\right), \mathcal{H}^{-1 *}\left(1_{z}\right)\right)\right)=0.7 . \\
0.9=\mathcal{H}\left(\top_{y}\right)(z) \neq \bigwedge_{x \in X}\left(\mathbf{T}_{H}\left(\mathcal{H}^{-1 *}\left(1_{x}\right)\right) \rightarrow\left(\mathcal{H}^{-1 *}\left(1_{x}\right)(y) \rightarrow \mathcal{H}^{-1 *}\left(1_{x}\right)(z)\right)\right) \\
=(0.5 \rightarrow(0.2 \rightarrow 0)) \wedge(0.7 \rightarrow(0.4 \rightarrow 0.5)) \wedge(0.7 \rightarrow(0.1 \rightarrow 0.3)) \\
=1
\end{gathered}
$$

Example 3.14. Let $\left(L=[0,1], \odot, \rightarrow{ }^{*}\right)$ be a complete residuated lattice as in Example 3.13. Let $X=\{x, y, z\}$ and $A, B \in L^{X}$ as follows:

$$
A(x)=0.9, A(y)=0.8, A(z)=0.3, B(x)=0.3, B(y)=0.7, B(z)=0.8
$$

Define $\mathcal{H}\left(1_{x}\right)(y)$ as follows

$$
\left(\begin{array}{ccc}
\mathcal{H}\left(1_{x}\right)(x)=1 & \mathcal{H}\left(1_{x}\right)(y)=0.8 & \mathcal{H}\left(1_{x}\right)(z)=0.6 \\
\mathcal{H}\left(1_{y}\right)(x)=0.7 & \mathcal{H}\left(1_{y}\right)(y)=1 & \mathcal{H}\left(1_{y}\right)(z)=0.3 \\
\mathcal{H}\left(1_{z}\right)(x)=0.5 & \mathcal{H}\left(1_{z}\right)(y)=0.6 & \mathcal{H}\left(1_{z}\right)(z)=1
\end{array}\right)
$$

(1) Since $\bigvee_{y \in X}\left(\mathcal{H}\left(1_{x}\right)(y) \odot \mathcal{H}\left(1_{y}\right)(z)=\mathcal{H}\left(\mathcal{H}\left(1_{x}\right)\right)(z)=\mathcal{H}\left(1_{x}\right)(z)\right.$ and $1_{x} \leq$ $\mathcal{H}\left(1_{x}\right)$ for all $x, y \in X$, then $\mathcal{H}$ is an upper approximation operator. Since $\mathcal{H}(A)(y)=\bigvee_{x \in X}\left(A(x) \odot\left(\mathcal{H}\left(1_{x}\right)(y)\right)\right.$, we have

$$
\mathcal{H}(A)=(0.9,0.8,0.5), \mathcal{H}(B)=(0.4,0.7,0.8) .
$$


Moreover, by Theorem 3.8,

$$
\left.\left.\mathbf{T}_{H}(A)=e_{L^{X}}(\mathcal{H}(A), A)\right)=0.8, \mathbf{T}_{H}(B)=e_{L^{X}}(\mathcal{H}(B), B)\right)=0.9 .
$$

(2) We obtain $\mathcal{H}^{-1}\left(1_{x}\right)(y)=\mathcal{H}\left(1_{y}\right)(x)$ as follows

$$
\left(\begin{array}{ccc}
\mathcal{H}^{-1}\left(1_{x}\right)(x)=1 & \mathcal{H}^{-1}\left(1_{x}\right)(y)=0.7 & \mathcal{H}^{-1}\left(1_{x}\right)(z)=0.5 \\
\mathcal{H}^{-1}\left(1_{y}\right)(x)=0.8 & \mathcal{H}^{-1}\left(1_{y}\right)(y)=1 & \mathcal{H}^{-1}\left(1_{y}\right)(z)=0.6 \\
\mathcal{H}^{-1}\left(1_{z}\right)(x)=0.6 & \mathcal{H}^{-1}\left(1_{z}\right)(y)=0.3 & \mathcal{H}^{-1}\left(1_{z}\right)(y)=1
\end{array}\right)
$$

Since $\bigvee_{y \in X}\left(\mathcal{H}^{-1}\left(1_{x}\right)(y) \odot \mathcal{H}^{-1}\left(1_{y}\right)(z)=\mathcal{H}^{-1}\left(1_{x}\right)(z)\right.$ and $1_{x} \leq \mathcal{H}^{-1}\left(1_{x}\right)$ for all $x, y \in X$, then $\mathcal{H}^{-1}$ is an upper approximation operator. Since $\mathcal{H}^{-1}(A)(y)=$ $\bigvee_{x \in X}\left(A(x) \odot\left(\mathcal{H}^{-1}\left(1_{x}\right)(y)\right)\right.$, we have

$$
\mathcal{H}^{-1}(A)=(0.9,0.8,0.4), \mathcal{H}^{-1}(B)=(0.5,0.7,0.8) .
$$

Moreover, by Theorem 3.8,

$$
\left.\left.\mathbf{T}_{H^{-1}}(A)=e_{L^{X}}\left(\mathcal{H}^{-1}(A), A\right)\right)=0.9, \mathbf{T}_{H^{-1}}(B)=e_{L^{X}}\left(\mathcal{H}^{-1}(B), B\right)\right)=0.8 .
$$

For $A^{*}=(0.1,0.2,0.7), \mathcal{H}^{-1}\left(A^{*}\right)=(0.3,0.2,0.7), \mathcal{H}\left(A^{*}\right)=(0.2,0.3,0.7)$ and

$$
\begin{gathered}
\mathbf{T}_{H^{-1}}(A)=\mathbf{T}_{H}\left(A^{*}\right)=e_{L^{X}}\left(\mathcal{H}\left(A^{*}\right), A^{*}\right)=0.9 \\
\mathbf{T}_{H}(A)=\mathbf{T}_{H^{-1}}\left(A^{*}\right)=e_{L^{X}}\left(\mathcal{H}^{-1}\left(A^{*}\right), A^{*}\right)=0.8 \\
\mathcal{H}\left(\mathcal{H}^{-1 *}\left(1_{x}\right)\right)=\mathcal{H}^{-1 *}\left(1_{x}\right)=(0,0.3,0.5) \\
\mathcal{H}\left(\mathcal{H}^{-1 *}\left(1_{y}\right)\right)=\mathcal{H}^{-1 *}\left(1_{y}\right)=(0.2,0,0.4) \\
\mathcal{H}\left(\mathcal{H}^{-1 *}\left(1_{z}\right)\right)=\mathcal{H}^{-1}\left(1_{z}\right)=(0.4,0.7,0) . \\
0.3=\mathcal{H}\left(\top_{y}\right)(z)=\bigwedge_{x \in X}\left(\mathbf{T}_{H}\left(\mathcal{H}^{-1 *}\left(1_{x}\right)\right) \rightarrow\left(\mathcal{H}^{-1 *}\left(1_{x}\right)(y) \rightarrow \mathcal{H}^{-1 *}\left(1_{x}\right)(z)\right)\right) \\
=(1 \rightarrow(0.3 \rightarrow 0.5)) \wedge(1 \rightarrow(0 \rightarrow 0.4)) \wedge(1 \rightarrow(0.7 \rightarrow 0)) .
\end{gathered}
$$

\section{References}

[1] R. Bělohlávek,Fuzzy Relational Systems, Kluwer Academic Publishers, New York , (2002), doi: 10.1007/978-1-4615-0633-1.

[2] P. Hájek, Metamathematices of Fuzzy Logic, Kluwer Academic Publishers, Dordrecht (1998), doi: 10.1007/978-94-011-5300-3. 
[3] Fang Jinming, I-fuzzy Alexandrov topologies and specialization orders, Fuzzy Sets and Systems, 158(2007), 2359-2374, doi: 10.1016/j.fss.2007.05.001.

[4] Y.C. Kim, Alexandrov $L$-topologies and $L$-join meet approximation operators International Journal of Pure and Applied Mathematics, 91(1)(2014), 113-129 doi: 10.12732/ijpam.v9lili.12.

[5] Y.C. Kim, Y.S. Kim, $L$-approximation spaces and $L$-fuzzy quasiuniform spaces, Information Sciences. 179(2009), 2028-2048, doi: 10.1016/j.ins.2009.02.003.

[6] J. Kortelainen, On relationships between modified sets, topological spaces and rough sets, Fuzzy Sets and Systems, 61(1994), 91-95, doi: 10.1016/0165-0114(94)90288-7.

[7] H. Lai, D. Zhang, Fuzzy preorder and fuzzy topology, Fuzzy Sets and Systems, 157 (2006), 1865-1885, doi: 10.1016/j.fss.2006.02.013.

[8] H. Lai, D. Zhang, Concept lattices of fuzzy contexts: Formal concept analysis vs. rough set theory, Int. J. Approx. Reasoning, 50 (2009), 695-707, doi: $10.1016 /$ j.ijar.2008.12.002.

[9] Z. Pawlak, Rough sets, Int. J. Comput. Inf. Sci., 11 (1982), 341-356, doi: 10.1007/BF01001956.

[10] Z. Pawlak, Rough probability, Bull. Pol. Acad. Sci. Math., 32(1984), 607615.

[11] A. M. Radzikowska, E.E. Kerre, A comparative study of fuzzy rough sets, Fuzzy Sets and Systems, 126(2002), 137-155, doi: 10.1016/so1650114(01)00032-x.

[12] S. P. Tiwari, A.K. Srivastava, Fuzzy rough sets, fuzzy preorders and fuzzy topologies, Fuzzy Sets and Systems, 210(2013), 63-68, doi: 10.1016/j.fss.2012.06.001.

[13] Q. Y. Zhang, L. Fan, Continuity in quantitive domains, Fuzzy Sets and Systems, 154 (2005), 118-131, doi: 10.1016/j.fss.2005.01.007.

[14] Q. Y. Zhang, W. X. Xie, Fuzzy complete lattices, Fuzzy Sets and Systems, 160(2009), 2275-2291, doi: 10.1016/j.fss.2008.12.001. 
[15] Y.H. She, G.J. Wang, An axiomatic approach of fuzzy rough sets based on residuated lattices, Computers and Mathematics with Applications, 58 (2009), 189-201, doi: 10.1016/j.camwa.2009.03.100.

[16] Zhen Ming Ma, Bao Qing Hu, Topological and lattice structures of Lfuzzy rough set determined by lower and upper sets, Information Sciences, 218(2013), 194-204, doi: 10.1016/j.ins.2012.06.029. 